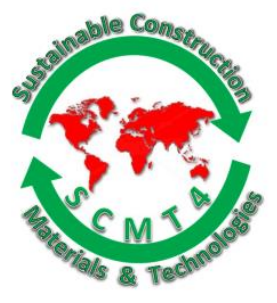

SCMT4

Las Vegas, USA, August 7-11, 2016

\title{
Effect of Supplemental Cementitious Materials on Electric Conductivity and Chloride Ingress of Hardened Cement Paste
}

\author{
Kiyfomi Kurumisawa ${ }^{1 a}$, and Toyoharu Nawa ${ }^{1 b}$ \\ ${ }^{I}$ Division of Sustainable Resources Engineering; Hokkaido University, Kital3 nishi8, Sapporo, Hokkaido, \\ Japan.1amail: <kurumi@eng.hokudai.ac.jp>, ${ }^{1 b}$ Email: <nawa@eng.hokudai.ac.jp >
}

\begin{abstract}
It is necessary to improve transport properties of concrete for lengthening the durability of concrete, therefore it is very important to measure the pore structure of hardened cement paste precisely in understanding mass transport properties. Measurement in electric conductivity by the AC impedance method is suggested as simple and easy technique to understand it, and the usefulness is reported in the case of hardened cement paste used with ordinary Portland cement. However, this technique has not been applied for hardened cement paste mixed with fly ash and blast furnace slag that has very high resistance to chloride ingress. In this study, chloride ingress and microstructure of the fly ash cement paste (FAC) and blast furnace slag cement paste (BFSC) was investigated by the electric conductivity measurement. For measuring the electric conductivity the AC impedance method was applied, and backscattered electron image analysis and mercury intrusion method was used for measuring pore structure in FAC and BFSC. The relationship between porosity and the electric conductivity was expressed by the Archie's law that is expressed with exponential function, and very high correlation was shown in chloride diffusion coefficient and electric conductivity regardless of fly ash and blast furnace slag replacement ratio.
\end{abstract}

\section{INTRODUCTION}

The durability of concrete is strongly influenced by the transport properties of the concrete, which may be described by its permeability and diffusion characteristics. Therefore, it is very important to be able to predict the transport properties of a concrete, and also to control the transport properties, in order to improve the characteristics, and to achieve this, reduction of the water to cement ratio, production of concrete with supplementary materials such as fly ash and blast furnace slag have been attempted. However, tests of the diffusion coefficient and water permeability to establish the transport properties of concrete are time consuming, especially case of blended cement concrete. It is known that the transport properties strongly depend on the pore structure, and that the diffusion coefficient is related to the porosity, tortuosity, and pore connectivity, and numerous methods for measurement of the pore structure have been suggested. A mercury intrusion method is widely used for measuring the pore structure. Backscattered electron image analysis (BEI) has also been performed to evaluate the pore structure at the submicron level in recent years [Scrivener 2004]. This technique could measure the quantity of pores in two-dimensional sections of samples, the quantity measured can be considered similar to that in three dimensions by performing a statistical analysis. However, it is impossible to evaluate the connectivity of the pores by BEI. 
To evaluate the connectivity of pores, electrochemical methods such as DC impedance or AC impedance measurements are conducted. About AC impedance measurements, it is possible to measure the electric conductivity from the bulk electric resistance of cementitious materials, and Christensen reported an investigation with the technique based on theory and experiments [Christensen 1994]. Numerous studies based on this technique were reported, and the effectiveness of measuring these properties of concrete has been shown [McCarter 1990; Keddam 1997; Ping 1993; Cabeza 2002; Sato 2011; Scuderi, 1991]. For the relationship between electric conductivity and properties of cement based materials, McCarter showed a good correlation between electric conductivity and permeability and the diffusion coefficient of mortar [McCarter 2000]. In addition, there are many studies connecting the electric conductivity between the properties of concrete [Andrade 1993; Streicher 1995; Sánchez 2011; Ortega 2012; Meilun 1999; Díaz 2006; Mercado 2012; Buenfeld 1987; Shane 2000; Vedalakshmi 2008; Narayanan 2010; Minagawa 2010]. However, most studies investigated limited ranges of water to cement ratios (W/C 0.4-0.6), the relationship between porosity and conductivity of mortar or concrete that includes the effect of the interfacial transition zone (ITZ) was established as shown in Table 1. Recently, high-strength concrete and high performance concrete that display a smaller effect of ITZ have been widely utilized [Vivekanandam 1997; Chen2004]. High strength concrete contains much cement matrix, and the performance of the cement matrix has a marked influence on the performance of the concrete. Therefore it is necessary to investigate hardened cement paste as cement matrix where there is no effect of ITZ. However, there are a few studies on hardened cement paste with a strong emphasis on the properties of concrete and mortar.

In this study, we measured the pore connectivity of hardened cement paste by the AC impedance method, the pore structure was measured by mercury intrusion porosimetry (MIP), and the purpose of this study is to clarify the relations among these. In addition, the relationship between the electric conductivity and the diffusion coefficient of the chloride ions in hardened cement paste with fly ash and blast furnace slag was also established. We evaluated the diffusivity of the chloride ions in hardened cement paste by evaluating the potential of the ability for mass transfer with an AC impedance method which is able to measure this parameter rapidly by a simple and easy setup. It is considered that this study is useful to understand the blended cement regarding pore structure.

\section{DIFFUSION AND ELECTRIC CONDUCTIVITY}

The diffusion flux of ion specie in porous media is expressed by the Nernst-Planck equation [Andrade 1993; Díaz 2006; Mercado 2012; Minagawa 2010],

$$
J_{i}=D_{e} \frac{\partial C_{i}}{\partial x}+\frac{z_{i} F}{R T} D_{e} C_{i} \frac{\partial \varphi}{\partial x}
$$

$J_{i}$ : the flux of an ion species $i, D_{e}$ : the effective diffusion coefficient of the ion species, $C$ : the concentration of the ion, $z$ : the valence number of the ion species, R: the gas constant, F: Faraday's constant, $\mathrm{T}$ : the temperature, $\varphi$ : the electrical field, and $\mathrm{x}$ : the position of medium. When there is no concentration gradient in a porous medium and the external potential is sufficiently large, equation (1) can be simplified as,

$$
J_{i}=\frac{z_{i} F}{R T} D_{e} C_{i} \frac{\partial \varphi}{\partial x}
$$

Then to convert the flux $J_{i}$ to the current density $I$, both sides of (2) are multiplied by $\mathrm{z}_{\mathrm{i}} \mathrm{F}$,

$$
I=z_{i} F J_{i}=\frac{z_{i}^{2} F^{2}}{R T} D_{e} C_{i} \frac{\partial \varphi}{\partial x}
$$

Now, the bulk electric conductivity $\sigma$ can be expressed by the following equation, 


$$
\sigma=\frac{I}{\left(\frac{\partial \varphi}{\partial x}\right)}
$$

Substituting for $I$ gives [Andrade 1993],

$$
\sigma=D_{e} \frac{z_{i}^{2} F^{2} C_{i}}{R T}
$$

This shows that the electric conductivity is proportional to the effective diffusion coefficient and the concentration of ions from equation (5). The effective diffusion coefficient $D_{e}$ and intrinsic diffusion coefficient $\mathrm{D}_{0}$ are related as

$$
D_{e}=\frac{\varepsilon}{\tau} S^{-m} D_{0}
$$

where $\varepsilon$ : the porosity, S:saturation (moisture condition), m: a constant, and $\tau$ : the tortuosity. Showing that the electric conductivity is also proportional to the porosity, saturation and tortuosity.

\section{EXPERIMENTAL INVESTIGATION}

Materials. Ordinary Portland cement (OPC) produced in Japan was used. Blast furnace slag (BFS) and fly ash (FA) were used for supplementaly materials. The water/cement ratios $(0.3,0.4,0.5,0.6$, and 0.7$)$ were used to produce a variety of microstructures in the hardened cement pastes for OPC. BFS repleacement ratio is $0.3,0.5$ and 0.7 for W/B $0.4,0.5$ for W/B 0.3 and 0.5 , and FA replacement ratio is 0.15 and 0.3 for W/B 0.4 specimens. Specimens with high water to cement ratio were mixed every 30 minute until bleeding water disappeared in order to avoid the effect of bleeding. The specimens were cast in $40 * 40 * 40 \mathrm{~mm}$ cubic molds for the electric conductivity measurements, and as $50 \mathrm{~mm}$ long $50 \mathrm{~mm}$ diameter cylinders for the chloride diffusion measurements. The specimens were demolded after $24 \mathrm{~h}$, and cured at $20{ }^{\circ} \mathrm{C}$ and $\mathrm{RH} .98 \%$ until prescribed age.

The electric conductivity. The size of specimens for the electric conductivity measurements was $40 * 40 * 40 \mathrm{~mm}$, the stainless electrodes $(40 * 30 * 0.3 \mathrm{~mm})$ were placed $30 \mathrm{~mm}$ apart on the specimen. The effective area of the electrode is $30 * 30 \mathrm{~mm}$, and the AC impedance of the specimens is measured in the range of $4 \mathrm{~Hz}$ to $5 \mathrm{MHz}$ with an impedance analyzer (HIOKI IM3570). After the measurements a Nyquist plot was established from the acquired data, and the bulk resistivity was determined from the point where the electrode resistivity (straight line) and an arc crossed.

$$
\begin{gathered}
\rho_{t}=\frac{R_{a} A}{L} \\
\sigma=\frac{1}{\rho_{t}}
\end{gathered}
$$

where, $\rho t$ : the bulk resistivity of specimen, Ra: the measured resistance, L: the distance between electrodes, A: the effective area of the electrode, and $\sigma$ : the conductivity. The electric conductivity of hardened cement paste depends on the porosity, pore connectivity, and conductivity of pore solution in the hardened cement paste.

\section{Chloride ingress depth measurement}

We measured the ingress depth of the chloride ions by an electron probe micro analyzer (EPMA: JEOL JXA-8900M). The specimens were $\varphi 50 * 50 \mathrm{~mm}$ (curing ages 28 and 91days) immersed in $\mathrm{NaCl} 0.5 \mathrm{~mol} / 1$ solution for 2 weeks, after that, the distribution of elements $(\mathrm{Ca}, \mathrm{Si}$, and $\mathrm{Cl}$ ) was determined by EPMA and the ingress depth of chloride was established. And apparent diffusion coefficient was determined by the 
following equation, with the concentration $C(x, t)$ derived from Fick's second law (initial and boundary conditions: $\mathrm{C}=\mathrm{C}_{0}$ at $\mathrm{x}=0, \mathrm{t}>0 ; \mathrm{C}=0$ at $\mathrm{x}>0, \mathrm{t}=0 ; \mathrm{C}=0$ at $\mathrm{x}=\infty, \mathrm{t}=0$ ),

$C(x, t)=C_{0} \cdot\left[1-\operatorname{erf}\left(\frac{x}{2 \sqrt{D_{a} \cdot t}}\right)\right]$

with erf: an error function, $\mathrm{x}$ : the distance from the exposed face, $\mathrm{t}$ : the exposure time, $\mathrm{C}_{0}$ : the bulk concentration, and $\mathrm{D}_{\mathrm{a}}$ : the apparent diffusion coefficient.

Mercury intrusion porosimetry. Mercury intrusion porosimetry (MIP) was used for masurement of pore size distribution. Crushed samples under 5mm sieve was used for MIP measurement.

\section{RESULTS AND DISCUSSIONS}

The electric conductivity. The results of electric conductivity measurements of the hardened cement pastes with various water to cement ratios, various replacement ratio of fly ash and BFSC are shown in figure 1. The electric conductivity of the specimens with different W/C specimens (figure 1(a)) became lower value with decreasing water to cement ratios, and the conductivity of all specimens became very similar after 7 days of curing. The electric conductivity of specimens mixed with BFS and FA decreased for a long term, the conductivity of blended cement paste were lower than that of OPC of the same water to binder ratio.

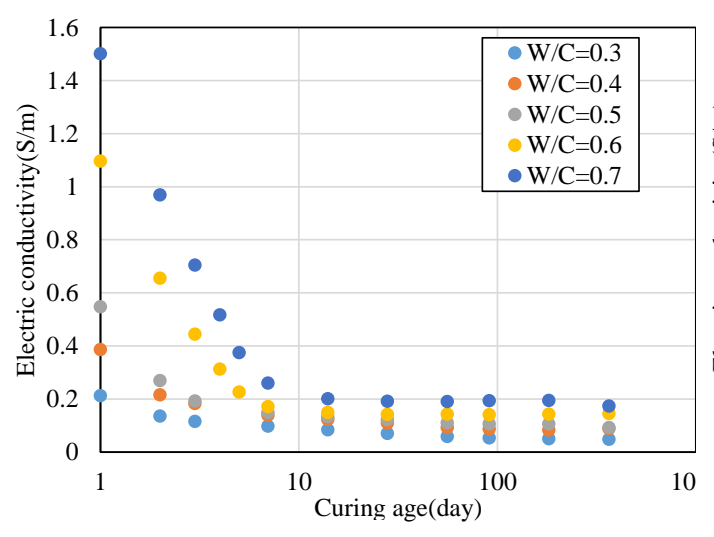

(a)

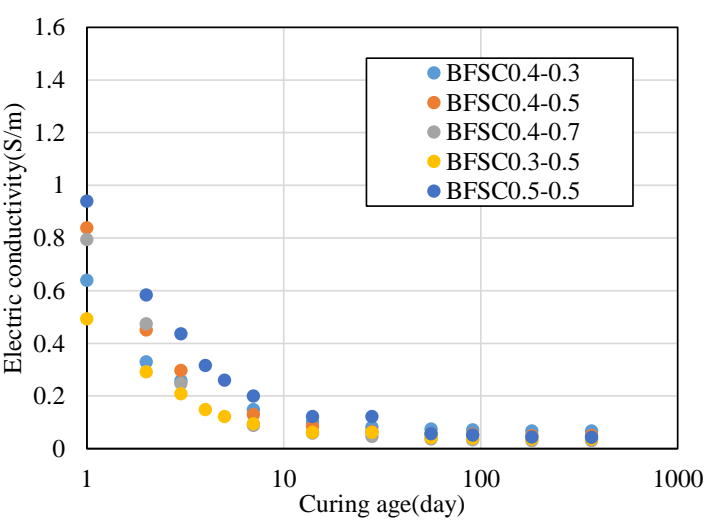

(b)

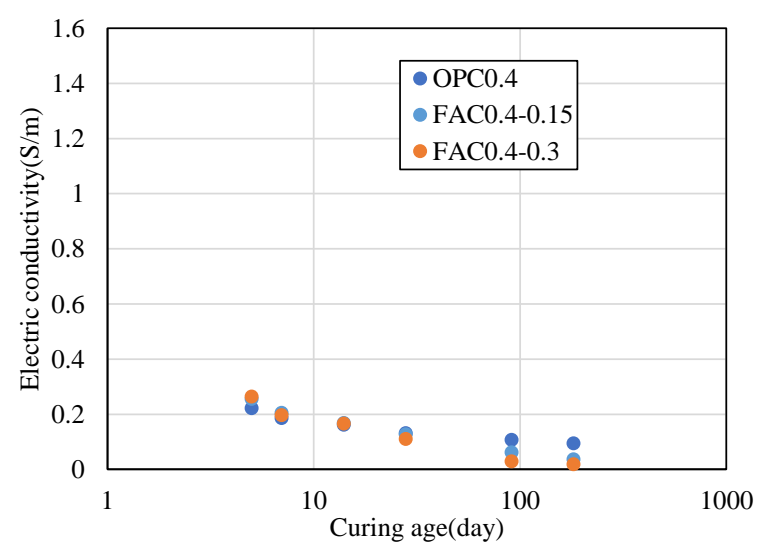

(c)

Figure 1. Electric conductivity of hardened cement pastes (a) OPC, (b) BFSC, (c) FAC. 
The electric conductivity, diffusion and pore structure. The effects of the pore volume measured by MIP on the conductivity of hardened cement pastes is shown in figure 2 . The conductivity decreased with a reduction of porosity by the MIP in all specimens. This relation was generally obeyed by the Archie's law, but the correlation coefficient of this relation was low in this study. In particular, for hardened cement pastes mixed with FA and BFS, a different tendency was shown. The effects of the pore volume measured by MIP on the apparent diffusion coefficient of hardened cement pastes is shown in figure 3. The diffusion coefficient of OPC pastes decreaseed with a reduction of porosity. However, the diffusivity of FAC and BFSC pastes did not decrease by a decrease in porosity. It was shown that other factors such as chloride adsorption or tortuosity shown in eqation.(6) influenced diffusivity as well as porosity.

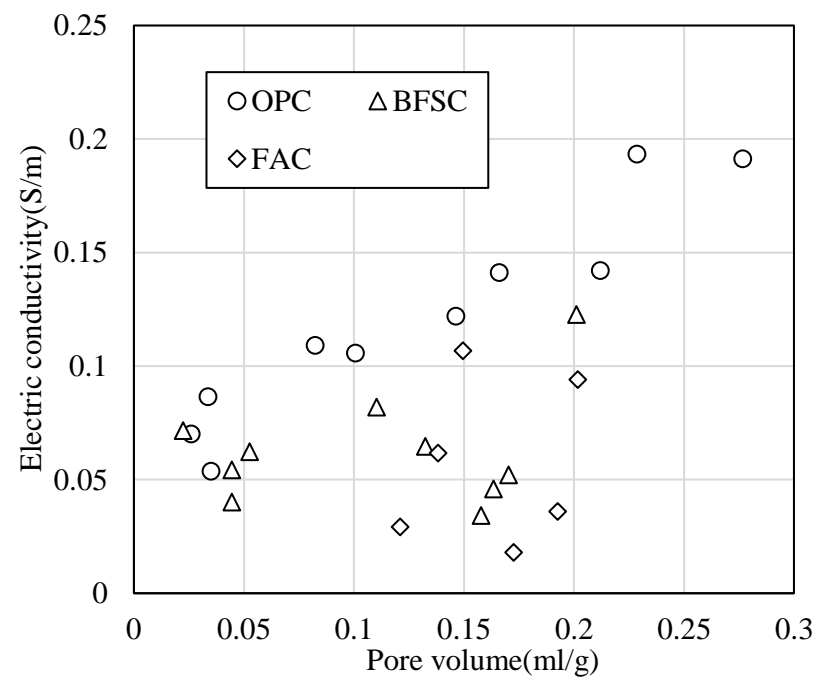

Figure 2. The effects of the pore volume measured by MIP on the conductivity of hardened

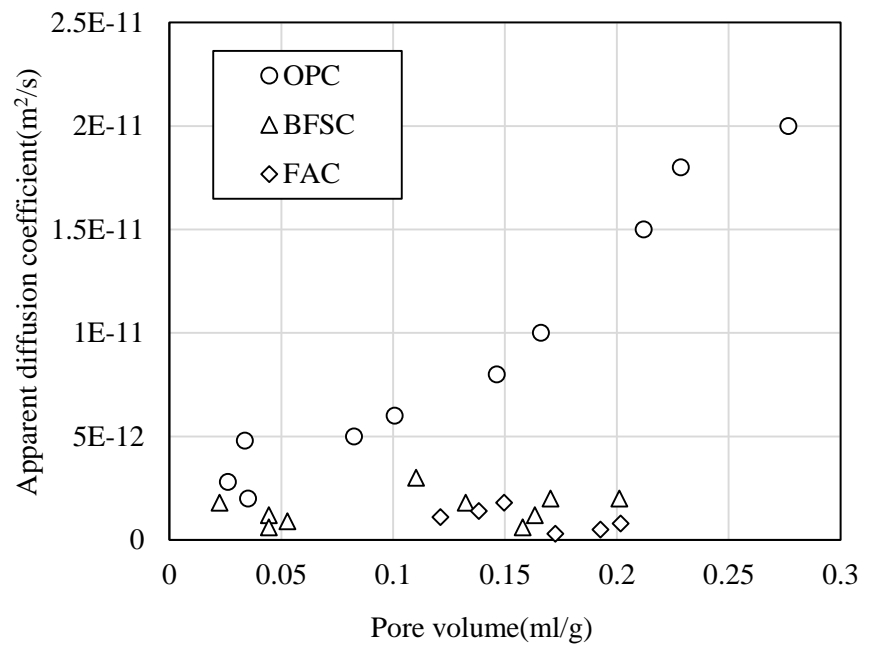

cement pastes

Figure 3. The effects of the pore volume measured by MIP on the apparent diffusion coefficient of hardened cement paste 
Chloride ingress and electric conductivity. The relationship between conductivity and apparent diffusion coefficient of chloride ions at 2 weeks immersion is shown in figure 4 . There is a very strong correlation between the electric conductivity and the diffusion coefficient of chloride ions in all of the specimens as shown in previous report [Atkinson 1984]. This result corresponds to the results of the concrete and mortar relationship between conductivity and diffusion coefficients in previous reports [Vedalakshmi 2008; Neithalath 2010; Minagawa 2010]. Although, it was shown that both the pore structure of the hardened cement paste and the composition of the pore solution influence the chloride ingress as shown in Eq.(6), the diffusion of ions is more strongly influenced by the pore structure. The effective diffusion coefficient has been shown to be associated with the apparent diffusion coefficient in other experiments [Yang 2005; Chiang 2007]. In summary it may be concluded that it is possible to predict the chloride ingress by measurement of the electric conductivity from these relations, and it is also possible to predict the durability of a new concrete structure, and evaluate the durability of existing concrete structures. However, it is necessary to consider the effect of the composition of pore solution and water content (moisture condition) in the concrete as shown in eq.(6) when the electric conductivity measurements are applied to evaluate the performance of in-situ concrete structures[Saleem 1996; Shi 2004; Kurumisawa 2013]. Therefore it is necessary to measure a moisture content in concrete before measuring an accurate electric conductivity on site.

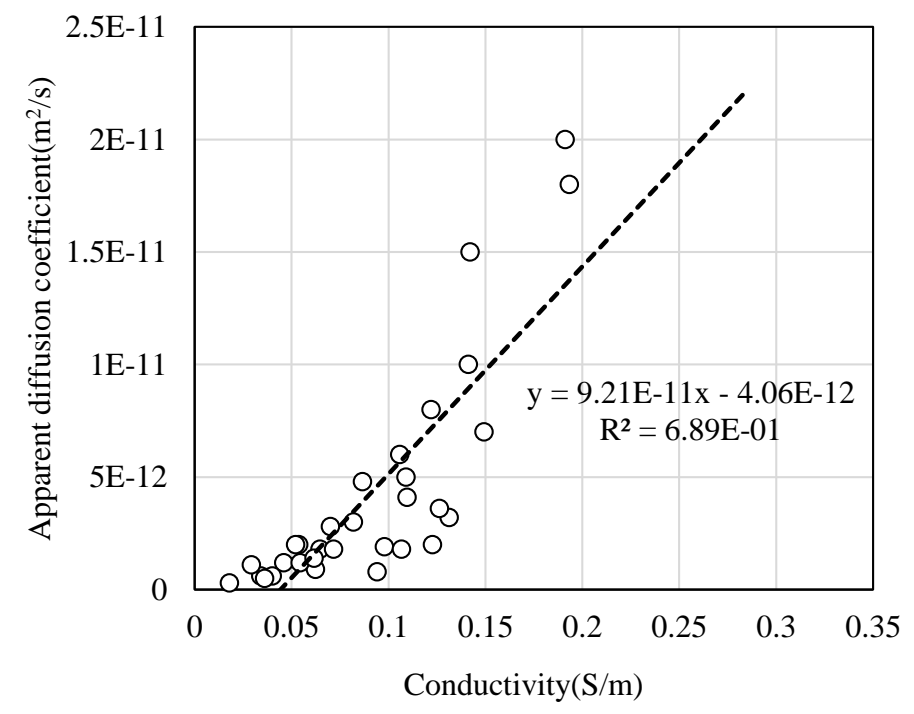

Figure 4. Plot of electric conductivity and apprent diffusion coefficient

\section{CONCLUSION}

The electric conductivity and microstructure of hardened cement paste with a range of water to cement ratios, different replacement ratio of fly ash and blast furnace slag was investigated in this study.

- The electric conductivity of hardened cement pastes depends on the porosity. These relations can be expressed by Archie's law in all specimens regardless of water to cement ratio, curing age, different replacement ratio of FA and BFS. However, the correlation between porosity and apparent diffusion coefficient was low.

- Finally, relations between the electric conductivity and the diffusion coefficient of chloride ions in hardened cement paste were established, and it was shown that it is possible to predict the chloride ingress by measurements of the electric conductivity. 


\section{ACKNOWLEDGEMENT}

We thank the Ministry of Education, Culture, Sports, Science and Technology for financial support with the research here.

\section{REFERENCES}

Andrade, C., (1993)."Calculation of chloride diffusion coefficients in concrete from ionic migration measurements", Cem. Concr. Res. 23 (3) 724-742.

Archie, G. E.: The electrical resistivity log as an aid in determining some reservoir characteristics, Trans. AIME 146 (1972) 54-62.

Atkinson, A., and Nickerson, A. K. (1984). "The diffusion of ions through water-saturated cement", J. Mater. Sci. 19 3068-3078

Buenfeld, N. Newman, J. (1987). "Examination of three methods for studying ion diffusion in cement pastes, mortars and concrete", Mater. Struct. 20(1) 3-10.

Cabeza, M., P. Merino, A. Miranda, X.R. Nóvoa, I. Sanchez, (2002). "Impedance spectroscopy study of hardened Portland cement paste", Cem. Concr. Res. 32(6) 881-891.

Chen, Bing., Juanyu Liu, (2004). "Effect of aggregate on the fracture behavior of high strength concrete", Constr. Build. Mater. 18(8) 585-590.

Chiang, C.T., Yang, C.C., (2007). "Relation between the diffusion characteristic of concrete from salt ponding test and accelerated chloride migration test", Mater. Chem. Phys. 106 240-246.

Christensen, Bruce J et al, (1994). "Impedance Spectroscopy of Hydrating Cement-Based Materials: Measurement, Interpretation", and Application, J. Am. Ceram. Soc. 772789-2804.

Díaz, B., X.R. Nóvoa, M.C. Pérez, (2006). "Study of the chloride diffusion in mortar: A new method of determining diffusion coefficients based on impedance measurements", Cem. Conc. Comp. 28(3) 237245.

Gu, Ping, Ping Xie, J.J. Beaudoin, R. Brousseau, (1993). “A.C. impedance spectroscopy (II): Microstructural characterization of hydrating cement-silica fume systems", Cem. Concr. Res. 23(1) 157-168.

Keddam, M., Takenouti, H., Nóvoa, X.R., Andrade, C., Alonso, C. (1997). "Impedance measurements on cement paste", Cem. Concr. Res. 27(8) 1191-1201.

Kurumisawa, K, and Nawa, T,. (2013). "Effect of microstructure and moisture content on the electric conductivity of hardened cement paste.", Proceeding of SCMT3.

Lu, Xinying(1997). "Application of the Nernst-Einstein Equation to Concrete", Cem. Concr. Res. 27(2) 293-302.

McCarter, W.J., Brousseau, R. (1990). “The A.C. response of hardened cement paste”, Cem. Concr. Res. 20(6) 891-900.

McCarter, W.J, Starrs, G., Chrisp, T.M, (2000). "Electric conductivity, diffusion, and permeability of Portland cement-based mortars", Cem. Concr. Res.30(9) 1395-1400.

Mercado, Hugo, Sylvie Lorente, Xavier Bourbon, (2012). "Chloride diffusion coefficient: A comparison between impedance spectroscopy and electrokinetic tests", Cem. Conc. Comp. 34(1) 68-75.

Minagawa, H. Hisada, M., Ehara, A., Saito, Y., Ichikawa, M., and Inoue, H. (2010). 'Fundamental study 
on relationship between electric resistivity and chloride ion diffusivity of concrete", Doboku Gakkai Ronbunshuu E. 66 (1) (in Japanese).

Neithalath, Narayanan, Jitendra Jain, (2010). "Relating rapid chloride transport parameters of concretes to microstructural features extracted from electric impedance", Cem. Concr. Res. 40(7) 1041-1051.

Ortega, J.M., I. Sánchez, M.A. Climent, (2012). "Durability related transport properties of OPC and slag cement mortars hardened under different environmental conditions", Constr. Build. Mater. 27(1) 176183.

Sánchez, I., M. P. López, J. M. Ortega, M. Á, (2011). "Impedance spectroscopy: An efficient tool to determine the non-steady-state chloride diffusion coefficient in building materials", Mater. Corros. 62(2) 139-145.

Saleem, M., Shameem, M., Hussain, S. E., \& Maslehuddin, M., (1996). "Effect of moisture, chloride and sulphate contamination on the electrical resistivity of Portland cement concrete." Construction and Building Materials, 10(3) 209-214.

Sato, Taijiro, Beaudoin, James, (2011). "Coupled AC impedance and thermomechanical analysis of freezing phenomena in cement paste", Mater. Struct. 44(2) 405-414.

Scrivener, Karen L. (2004). "Backscattered electron imaging of cementitious microstructures: understanding and quantification", Cem. Conc. Comp. 26 935-945.

Scrivener, K.L., T. Füllmann, E. Gallucci, G. Walenta, E. Bermejo, (2004). "Quantitative study of Portland cement hydration by X-ray diffraction/Rietveld analysis and independent methods", Cem. Concr. Res. 34 1541-1547.

Scuderi, C. A., Mason, T. O., Jennings, H. M., (1991). "Impedance spectra of hydrating cement pastes", J. Mater. Sci. 26(2) 349-353.

Shi, C., (2004) . "Effect of mixing proportions of concrete on its electrical conductivity and the rapid chloride permeability test (ASTM C1202 or ASSHTO T277) results.”, Cement and Concrete Research. 34(3) 537-545.

Shi, Meilun, Zhiyuan Chen, Jian Sun, (1999). "Determination of chloride diffusivity in concrete by AC impedance spectroscopy”, Cem. Concr. Res. 29(7) 1111-1115.

Shane, John D. Mason, Thomas O. Jennings, Hamlin M. Garboczi, Edward J. Bentz, Dale P. (2000). "Effect of the Interfacial Transition Zone on the Conductivity of Portland Cement Mortars", J. Am. Ceram. Soc. 83(5) 1137-1144.

Stauffer, D., and A. Aharony, (1992). "Introduction to Percolation Theory" (Taylor \& Francis, London).

Streicher, P.E., M.G. Alexander, (1995). "A chloride conduction test for concrete", Cem. Concr. Res. 25(6) 1284-1294.

Vedalakshmi, R., R. Renugha Devi, E. Bosco, N. Palaniswamy, (2008). "Determination of diffusion coefficient of chloride in concrete: an electrochemical impedance spectroscopic approach", Mater Struct, 41 1315-1326.

Vivekanandam, Kangesu, Indubhushan Patnaikuni, (1997). "Transition zone in high performance concrete during hydration”, Cem. Concr. Res. 27(6) 817-823.

Yang, C.C., (2005). "Acomparison of transport properties for concrete using ponding test and the accelerated chloride migration test", Mater. Struct. 38 313-320. 